\title{
A Generalization and Study of New Mock Theta Functions
}

\author{
Bhaskar Srivastava
}

Department of Mathematics and Astronomy, Lucknow University, Lucknow, India

\begin{abstract}
Two sets of mock theta functions were developed, one by Andrews and the other by Bringmann et al. We have given two generalizations and shown they belong to the class of $F_{q}$-functions. Relations between these generalized functions is established. Later we give $q$-Integral representation and multibasic expansions of these generalized $F_{q}$-functions.
\end{abstract}

Keywords Mock theta function, $q$-Multibasic expansion and $q$-Integral

\section{Brief History of Mock Theta Functions}

Ramanujan in his last letter to Hardy, dated January 12, 1920 , to be specific [18, pp. 354-355] gave a list of seventeen functions which he called "mock theta functions". The functions are of a complex variable $q$ defined by a $q$-series convergent for $|q|<1$. As $q$ approaches a root of unity. Ramanujan stated, they have certain asymptotic properties, similar to the properties of theta functions, but he conjectured that they are not theta functions. He also stated some identities relating some of the functions to each other. The list was divided into four group of functions of order three, five, five and seven. Watson[23] studied the third order mock theta functions and introduced three new one. Watson proved that the third order functions have asymptotic properties, as stated by Ramanujan and also that they are not theta functions. Watson proved the asymptotic formula, for the fifth order mock theta functions and Selberg for the seventh order mock theta functions, but neither author proved that the functions are not theta functions.

In 1976, Andrews discovered "Lost" Notebook while visiting Trinity College, Cambridge in the mathematical library of the college, written by Ramanujan towards the end of his life. In the "lost" notebook were six more mock theta functions and linear relations between them, Andrews and Hickerson [6] called them of sixth order. On the page 9 of the "Lost" Notebook appears four more mock theta functions, which were called by Choi [9] of tenth order.

Gordon and McIntosh, listed eight functions and called them of eighth order. Later in their survey paper [12] called only four functions of eighth order, the other four were of lower order. Hikami [13] in his work on Mathematical

* Corresponding author:

bhaskarsrivastav@yahoo.com (Bhaskar Srivastava)

Published online at http://journal.sapub.org/ajcam

Copyright (C) 2012 Scientific \& Academic Publishing. All Rights Reserved
Physics Quantum Invariant of three manifold came across a mock theta function and called it of second order.

Recently in his path breaking paper [5] while studying the $q$-orthogonal polynomials found some new mock theta functions. The following two mock theta functions are interesting

$$
\begin{aligned}
& \bar{\psi}_{0}(q)=\sum_{n=0}^{\infty} \frac{q^{2 n^{2}}}{(-q ; q)_{2 n}}=\frac{1}{\left(q^{2} ; q^{2}\right)_{\infty}} \sum_{n=0}^{\infty} q^{4 n^{2}+n}\left(1-q^{6 n+3}\right) \sum_{j=-n}^{n}(-1)^{j} q^{-j^{2}}, \\
& \bar{\psi}_{1}(q)=\sum_{n=0}^{\infty} \frac{q^{2 n^{2}+2 n}}{(-q ; q)_{2 n+1}}=\frac{1}{\left(q^{2} ; q^{2}\right)_{\infty}} \sum_{n=0}^{\infty} q^{4 n^{2}+3 n}\left(1-q^{2 n+1}\right) \sum_{j=-n}^{n}(-1)^{j} q^{-j^{2}}, \\
& \bar{\psi}_{2}(q)=\sum_{n=0}^{\infty} \frac{q^{2 n^{2}+2 n}\left(q ; q^{2}\right)_{n}}{\left(q^{2} ; q^{2}\right)_{n}(-q ; q)_{2 n}}=\frac{1}{\left(q^{2} ; q^{2}\right)_{\infty}} \sum_{n=0}^{\infty} q^{4 n^{2}+2 n}\left(1-q^{4 n+2}\right) \sum_{j=-n}^{n}(-1)^{j} q^{-j^{2}}, \\
& \text { and }
\end{aligned}
$$

$\bar{\psi}_{3}(q)=\sum_{n=0}^{\infty} \frac{q^{n^{2}}(-q ; q)_{n}^{2}}{(q ; q)_{2 n}}=\frac{1}{(q ; q)_{\infty}} \sum_{n=0}^{\infty} q^{2 n^{2}+n}\left(1-q^{6 n+6}\right) \sum_{j=0}^{n} q^{-\left(\begin{array}{l}j+1 \\ 2\end{array}\right)}$.

Bringmann, Hikami and Lovejoy [8] also found two more new mock theta functions

$$
\bar{\phi}_{0}(q)=\sum_{n=0}^{\infty} q^{n}(-q)_{2 n+1}
$$

and

$$
\bar{\phi}_{1}(q)=\sum_{n=0}^{\infty} q^{n}(-q)_{2 n} .
$$

In this paper we have given two generalizations of these mock theta functions and have shown they belong to the family of $F_{q}$-functions. Being $F_{q}$-functions they have unified properties, for example:

(i) The inverse operator $D_{q, x}^{-1}$ of $q$-differentiation is related to $q$-integration as

$$
D_{q, x}^{-1} f(x)=(1-q)^{-1} \int f(x) d_{q}(x)
$$

(ii) $D_{q, z}^{n} F(z, \alpha)=F(z, \alpha+n)$, where $n$ is a non-negative integer.

The scheme of the paper is as follows: 
We define a $F_{q}$-function in section 3 .

The definition of one generalization of these mock theta functions is in section 4 .

Section 5 contains relations between these generalized functions.

In section 6 we define a second generalization of these functions and show they are $F_{q}$-functions.

Section 7 contains more relations between these generalized functions.

In section 8 the generalized $F_{q}$-functions are represented as $q$-Integrals.

In section 9, multibasic expansions are given for these generalized $F_{q}$-functions.

\section{Basic Facts}

We shall use the following usual basic hypergeometric notations :

For $\left|q^{k}\right|<1$,

$$
\begin{aligned}
& \left(a ; q^{k}\right)_{n}=(1-a)\left(1-a q^{k}\right) \ldots\left(1-a q^{k(n-1)}\right), n \geq 1 \\
& \left(a ; q^{k}\right)_{0}=1 \\
& \left(a ; q^{k}\right)_{\infty}=\prod_{j=0}^{\infty}\left(1-a q^{k j}\right) .
\end{aligned}
$$

For convenience we shall write

$$
\left(a_{1}, a_{2}, \ldots \ldots, a_{m} ; q^{k}\right)_{n}=\left(a_{1} ; q^{k}\right)_{n}\left(a_{2} ; q^{k}\right)_{n} \ldots \ldots\left(a_{m} ; q^{k}\right)_{n} .
$$

When $k=1$, we usually write $(a)_{n}$ and $(a)_{\infty}$ instead of $(a ; q)_{n}$ and $(a ; q)_{\infty}$, respectively.

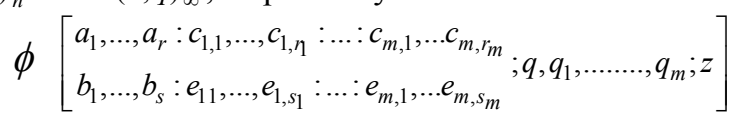

$$
\begin{aligned}
& =\sum_{n=0}^{\infty} \frac{\left(a_{1} \ldots \ldots, a_{r} ; q\right)_{n}}{\left(q, b_{1}, \ldots \ldots, b_{s} ; q\right)_{n}} z^{n}\left[(-1)^{\frac{n^{2}-n}{2}}\right]^{\frac{n^{2}}{2}} \prod_{j=1}^{1+s-r} \frac{\left(c_{j, 1}, \ldots \ldots ., c_{j, r_{j}} ; q_{j}\right)_{n}}{\left(e_{j, 1}, \ldots \ldots, e_{j, s_{j}} ; q_{j}\right)_{n}}\left[(-1)^{n} q_{j}^{\frac{n^{2}-n}{2}}\right]^{s_{j}-r_{j}} .
\end{aligned}
$$

A generalized basic hypergeometric series with base $q_{1}$ is defined as

$$
\begin{aligned}
& { }_{A} \varphi_{A-1}\left[a_{1}, a_{2}, \ldots, a_{A} ; b_{1}, b_{2} \ldots, b_{A-1} ; q_{1}, z\right] \\
= & \sum_{n=0}^{\infty} \frac{\left(a_{1} ; q_{1}\right)_{n} \ldots .\left(a_{A} ; q_{1}\right)_{n} z^{n}}{\left(b_{1} ; q_{1}\right)_{n} \ldots .\left(b_{A-1} ; q_{1}\right)_{n}\left(q_{1} ; q_{1}\right)_{n}},|\mathrm{z}|<1 .
\end{aligned}
$$

\section{Definition of $F_{q}$-Functions}

Truesdell [22] in his book, "Unified theory of special functions" calls a function $F$-function, if it satisfies the functional equation

$$
\frac{\partial}{\partial z} F(z, \alpha)=F(z, \alpha+1) .
$$

The $q$-analogue is: A function is called $F_{q}$-Function if it satisfies the functional equation

where

$$
D_{q, z} F(z, \alpha)=F(z, \alpha+1),
$$

$$
{ }_{z} D_{q, z} \quad F(z, \alpha)=F(z, \alpha)-F(z q, \alpha) .
$$

\section{Generalization of Mock Theta Func- tions and are $F_{q}$-Functions}

We give a generalization of these mock theta functions and show they are $F_{q}$-functions.

Definition of the generalized functions:

$$
\begin{aligned}
& \bar{\psi}_{0}(t, \alpha, q)=\frac{1}{(t)_{\infty}} \sum_{n=0}^{\infty} \frac{(t)_{n} q^{2 n^{2}-n+n \alpha}}{(-q ; q)_{2 n}}, \\
& \bar{\psi}_{1}(t, \alpha, q)=\frac{1}{(t)_{\infty}} \sum_{n=0}^{\infty} \frac{(t)_{n} q^{2 n^{2}+n+n \alpha}}{(-q ; q)_{2 n+1}}, \\
& \bar{\psi}_{2}(t, \alpha, q)=\frac{1}{(t)_{\infty}} \sum_{n=0}^{\infty} \frac{(t)_{n} q^{2 n^{2}+n+n \alpha}\left(q ; q^{2}\right)_{n}}{\left(q^{2} ; q^{2}\right)_{n}(-q ; q)_{2 n}}, \\
& \bar{\psi}_{3}(t, \alpha, q)=\frac{1}{(t)_{\infty}} \sum_{n=0}^{\infty} \frac{(t)_{n} q^{n^{2}-n+n \alpha}(-q ; q)_{n}}{(q ; q)_{n}\left(q^{1 / 2} ; q\right)_{n}\left(-q^{1 / 2} ; q\right)_{n}}, \\
& \bar{\phi}_{0}(t, \alpha, q)=\frac{1}{(t)_{\infty}} \sum_{n=0}^{\infty}(t)_{n} q^{n+n \alpha}(-q ; q)_{2 n+1},
\end{aligned}
$$

and

$$
\bar{\phi}_{1}(t, \alpha, q)=\frac{1}{(t)_{\infty}} \sum_{n=0}^{\infty}(t)_{n} q^{n+n \alpha}(-q ; q)_{2 n} .
$$

For $t=0$ and $\alpha=1$, the generalized functions defined in (4.1)-(4.4) reduce to mock theta functions $\bar{\psi}_{0}(q), \bar{\psi}_{1}(q)$ $\bar{\psi}_{2}(q)$ and $\bar{\psi}_{3}(q)$, respectively. For $t=0, \alpha=0$ the generalized functions defined in (4.5)-(4.6) reduce to the mock theta functions $\bar{\phi}_{0}(q)$ and $\bar{\phi}_{1}(q)$, respectively.

\section{Theorem 1}

$\bar{\psi}_{0}(t, \alpha, q), \bar{\psi}_{1}(t, \alpha, q), \quad \bar{\psi}_{2}(t, \alpha, q), \quad \bar{\psi}_{3}(t, \alpha, q), \bar{\phi}_{0}(t, \alpha, q)$ and $\bar{\phi}_{1}(t, \alpha, q)$ are $F_{q}$-functions.

We shall give the proof for $\bar{\psi}_{0}(t, \alpha, q)$ only. The proofs for the other functions are similar, hence omitted.

\section{Proof}

Applying the difference operator $D_{q, t}$, we have

$$
\begin{aligned}
t D_{q, t} & \bar{\psi}_{0}(t, \alpha, . q)=\bar{\psi}_{0}(t, \alpha, q)-\bar{\psi}_{0}(t q, \alpha, q) \\
& =\frac{1}{(t)_{\infty}} \sum_{n=0}^{\infty} \frac{(t)_{n} q^{2 n^{2}-n+n \alpha}}{(-q ; q)_{2 n}}-\frac{1}{(t q)_{\infty}} \sum_{n=0}^{\infty} \frac{(t q)_{n} q^{22^{2}-n+n \alpha}}{(-q ; q)_{2 n}} \\
& =\frac{1}{(t)_{\infty}} \sum_{n=0}^{\infty} \frac{(t)_{n} q^{2 n^{2}-n+n \alpha}}{(-q ; q)_{2 n}}-\frac{1}{(t)_{\infty}} \sum_{n=0}^{\infty} \frac{(t)_{n} q^{2 n^{2}-n+n \alpha}\left(1-t q^{n}\right)}{(-q ; q)_{2 n}} \\
& =\frac{t}{(t)_{\infty}} \sum_{n=0}^{\infty} \frac{(t)_{n} q^{22^{2}-n+n(\alpha+1)}}{(-q ; q)_{2 n}} .
\end{aligned}
$$

So

$$
D_{q, t} \bar{\psi}_{0}(t, \alpha, q)=\bar{\psi}_{0}(t, \alpha+1, q)
$$

Hence $\bar{\psi}_{0}(t, \alpha, q)$ is a $F_{q}$-function. By similar working all the generalized functions given in Theorem 1 are $F_{q}$ -functions. 


\section{Relation between the Generalized $F_{q}$ -functions $\bar{\psi}_{0}(t, \alpha, q), \bar{\psi}_{1}(t, \alpha, q)$ and $\bar{\phi}_{0}(t, \alpha, q), \bar{\phi}_{1}(t, \alpha, q)$}

Theorem 2

$$
D_{q, t}^{2} \bar{\psi}_{0}(t, \alpha, q)=\bar{\psi}_{1}(t, \alpha, q)+q D_{q, t}^{2} \bar{\psi}_{1}(t, \alpha, q) .
$$

\section{Proof}

$$
\begin{aligned}
& \text { Now } \begin{aligned}
D_{q, t}^{2} \bar{\psi}_{0}(t, \alpha, q)=\bar{\psi}_{0}(t, \alpha+2, q) \\
=\frac{1}{(t)_{\infty}} \sum_{n=0}^{\infty} \frac{(t)_{n} q^{2 n^{2}-n+n(\alpha+2)}}{(-q ; q)_{2 n}} \\
=\frac{1}{(t)_{\infty}} \sum_{n=0}^{\infty} \frac{(t)_{n} q^{2 n^{2}+n+n \alpha}}{(-q ; q)_{2 n+1}}\left(1+q^{2 n+1}\right) \\
=\frac{1}{(t)_{\infty}} \sum_{n=0}^{\infty} \frac{(t)_{n} q^{2 n^{2}+n+n \alpha}}{(-q ; q)_{2 n+1}}+\frac{q}{(t)_{\infty}} \sum_{n=0}^{\infty} \frac{(t)_{n} q^{2 n^{2}+3 n+n \alpha}}{(-q ; q)_{2 n+1}} \\
=\bar{\psi}_{1}(t, \alpha, q)+q D_{q, t}^{2} \bar{\psi}_{1}(t, \alpha, q),
\end{aligned}
\end{aligned}
$$

which proves Theorem 2 .

Theorem 3

$$
\bar{\phi}_{0}(t, \alpha, q)=\bar{\phi}_{1}(t, \alpha, q)+q D_{q, t}^{2} \bar{\phi}_{1}(t, \alpha, q) .
$$

\section{Proof}

By definition

$$
\begin{aligned}
& \bar{\phi}_{0}(t, \alpha, q)=\frac{1}{(t)_{\infty}} \sum_{n=0}^{\infty}(t)_{n} q^{n+n \alpha}(-q ; q)_{2 n+1} \\
= & \frac{1}{(t)_{\infty}} \sum_{n=0}^{\infty}(t)_{n} q^{n+n \alpha}\left(1+q^{2 n+1}\right)(-q ; q)_{2 n} \\
= & \frac{1}{(t)_{\infty}} \sum_{n=0}^{\infty}(t)_{n} q^{n+n \alpha}(-q ; q)_{2 n}+\frac{1}{(t)_{\infty}} \sum_{n=0}^{\infty}(t)_{n} q^{3 n+n \alpha+1}(-q ; q)_{2 n} \\
= & \bar{\phi}_{1}(t, \alpha, q)+q D_{q, t}^{2} \bar{\phi}_{1}(t, \alpha, q),
\end{aligned}
$$$$
\text { which proves Theorem } 3 \text {. }
$$

\section{Another Generalization of $\bar{\psi}_{0}(q)$, $\overline{\psi_{1}}(q)$ and $\operatorname{are} F_{q}$-functions}

We now give another generalization for $\bar{\psi}_{0}(q)$, $\bar{\psi}_{1}(q)$ and define :

$$
\bar{\psi}_{0}(t, z, q)=\frac{1}{(t)_{\infty}} \sum_{n=0}^{\infty} \frac{(t)_{n} q^{2 n^{2}} z^{4 n}}{(-q ; q)_{2 n}},
$$

and

$$
\bar{\psi}_{1}(t, z, q)=\frac{1}{(t)_{\infty}} \sum_{n=0}^{\infty} \frac{(t)_{n} q^{2 n^{2}+2 n} z^{4 n+2}}{(-q ; q)_{2 n+1}} .
$$

If we put $t=0, z=1$ in (6.1) and (6.2), the generalized functions reduce to mock theta functions $\bar{\psi}_{0}(q)$ and $\bar{\psi}_{1}(q)$ respectively.

By taking $z=q^{\alpha / 8}$ in (6.1) and (6.2), it can be shown, as is done in section 4 , that they are $F_{q}$-functions.

\section{Relations between Generalized Functions}

\section{Theorem 4}

$$
\bar{\psi}_{0}(t, \sqrt{z}, q)+\bar{\psi}_{0}(t, \sqrt{z q}, q)=\frac{2}{(t)_{\infty}}+z q \bar{\psi}_{1}(t q, \sqrt{z q}, q)
$$

and

$$
\bar{\psi}_{1}(t, \sqrt{z}, q)+\bar{\psi}_{1}(t, \sqrt{z q}, q)=\mathrm{z} \bar{\psi}_{0}(t, \sqrt{z q}, q)
$$

Proof

Writing for $z^{\frac{1}{2}}$ for $z$ in (6.1) and (6.2), we have

$$
\bar{\psi}_{0}(t, \sqrt{z}, q)=\frac{1}{(t)_{\infty}} \sum_{n=0}^{\infty} \frac{(t)_{n} q^{2 n^{2}} z^{2 n}}{(-q ; q)_{2 n}}
$$

and

$$
\bar{\psi}_{1}(t, \sqrt{z}, q)=\frac{1}{(t)_{\infty}} \sum_{n=0}^{\infty} \frac{(t)_{n} q^{2 n^{2}+2 n} z^{2 n+1}}{(-q ; q)_{2 n+1}} .
$$

So

$$
\begin{aligned}
\bar{\psi}_{0}(t, \sqrt{z}, q) & +\bar{\psi}_{0}(t, \sqrt{z q}, q)=\frac{1}{(t)_{\infty}} \sum_{n=0}^{\infty} \frac{(t)_{n} q^{2 n^{2}} z^{2 n}}{(-q ; q)_{2 n}}+\frac{1}{(t)_{\infty}} \sum_{n=0}^{\infty} \frac{(t)_{n} q^{2 n}}{(-q ; q)} \\
= & \frac{1}{(t)_{\infty}}\left[2+\sum_{n=1}^{\infty}\left\{\frac{(t)_{n} q^{2 n^{2}} z^{2 n}}{(-q ; q)_{2 n}}+\frac{(t)_{n} q^{2 n^{2}+2 n} z^{2 n}}{(-q ; q)_{2 n}}\right\}\right] \\
= & \frac{1}{(t)_{\infty}}\left[2+\sum_{n=1}^{\infty} \frac{(t)_{n} q^{2 n^{2}} z^{2 n}}{(-q ; q)_{2 n-1}}\right] \\
= & \frac{1}{(t)_{\infty}}\left[2+\sum_{n=0}^{\infty} \frac{(t)_{n+1} q^{2 n^{2}+4 n+2} z^{2 n+2}}{(-q ; q)_{2 n+1}}\right] \\
= & \frac{2}{(t)_{\infty}}+\frac{z q}{(t q)_{\infty}} \sum_{n=0}^{\infty} \frac{(t q)_{n} q^{2 n^{2}+2 n}(z q)^{2 n+1}}{(-q ; q)_{2 n+1}} \\
= & \frac{2}{(t)_{\infty}}+z q \bar{\psi}_{1}(t q, \sqrt{z q}, q),
\end{aligned}
$$

which is (7.1).

Again

$$
\begin{aligned}
\bar{\psi}_{1}(t, \sqrt{z}, q)+\bar{\psi}_{1}(t, \sqrt{z q}, q)= & \frac{1}{(t)_{\infty}} \sum_{n=0}^{\infty} \frac{(t)_{n} q^{2 n^{2}+2 n} z^{2 n+1}}{(-q ; q)_{2 n+1}} \\
& +\frac{1}{(t)_{\infty}} \sum_{n=0}^{\infty} \frac{(t)_{n} q^{2 n^{2}+4 n+1} z^{2 n+1}}{(-q ; q)_{2 n+1}} \\
= & \frac{1}{(t)_{\infty}} \sum_{n=0}^{\infty} \frac{(t)_{n} q^{2 n^{2}+2 n}\left(1+q^{2 n+1}\right) z^{2 n+1}}{(-q ; q)_{2 n+1}} \\
= & \frac{z}{(t)_{\infty}} \sum_{n=0}^{\infty} \frac{(t)_{n} q^{2 n^{2}}(z q)^{2 n}}{(-q ; q)_{2 n}} \\
= & z \bar{\psi}_{0}(t, \sqrt{z q}, q),
\end{aligned}
$$

which is (7.2). 


\section{Theorem 5}

$$
\bar{\psi}_{0}(t, i z, q)=\bar{\psi}_{0}(t, z, q)
$$

and

$$
\bar{\psi}_{1}(t, i z, q)=-\bar{\psi}_{1}(t, z, q)
$$

Proof

$$
\begin{aligned}
\bar{\psi}_{0}(t, i z, q) & =\frac{1}{(t)_{\infty}} \sum_{n=0}^{\infty} \frac{(t)_{n} q^{2 n^{2}} z^{4 n}}{(-q ; q)_{2 n}} \\
& =\bar{\psi}_{0}(t, z, q),
\end{aligned}
$$

which is $(7.3)$.

Again

$$
\bar{\psi}_{1}(t, i z, q)=-\frac{1}{(t)_{\infty}} \sum_{n=0}^{\infty} \frac{(t)_{n} q^{2 n^{2}+2 n} z^{4 n+2}}{(-q ; q)_{2 n+1}}
$$$$
=-\bar{\psi}_{1}(t, z, q)
$$

which is (7.4).

\section{8. $q$-Integral Representation for the Generalized $F_{q}$-functions} 19] as

The $q$-integral was defined by Thomae and Jackson [11, p.

$$
\int_{0}^{1} f(t) d_{q} t=(1-q) \sum_{n=0}^{\infty} f\left(q^{n}\right) q^{n}
$$

\section{Theorem 6}

(i) $\bar{\psi}_{0}\left(q^{t}, \alpha, q\right)=\frac{(1-q)^{-1}}{(q ; q)_{\infty}} \int_{0}^{1} w^{t-1}(w q ; q)_{\infty} \bar{\psi}_{0}(0, a w, q) d_{q} w$,

(ii) $\bar{\psi}_{1}\left(q^{t}, \alpha, q\right)=\frac{(1-q)^{-1}}{(q ; q)_{\infty}} \int_{0}^{1} w^{t-1}(w q ; q)_{\infty} \bar{\psi}_{1}(0, a w, q) d_{q} w$,

(iii) $\bar{\psi}_{2}\left(q^{t}, \alpha, q\right)=\frac{(1-q)^{-1}}{(q ; q)_{\infty}} \int_{0}^{1} w^{t-1}(w q ; q)_{\infty} \bar{\psi}_{2}(0, a w, q) d_{q} w$

(iv) $\bar{\psi}_{3}\left(q^{t}, \alpha, q\right)=\frac{(1-q)^{-1}}{(q ; q)_{\infty}} \int_{0}^{1} w^{t-1}(w q ; q)_{\infty} \bar{\psi}_{3}(0, a w, q) d_{q} w$

(v) $\bar{\phi}_{0}\left(q^{t}, \alpha, q\right)=\frac{(1-q)^{-1}}{(q ; q)_{\infty}} \int_{0}^{1} w^{t-1}(w q ; q)_{\infty} \bar{\phi}_{0}(0, a w, q) d_{q} w$,

(vi) $\bar{\phi}_{1}\left(q^{t}, \alpha, q\right)=\frac{(1-q)^{-1}}{(q ; q)_{\infty}} \int_{0}^{1} w^{t-1}(w q ; q)_{\infty} \bar{\phi}_{1}(0, a w, q) d_{q} w$.

\section{Proof}

We give a detailed proof of Theorem 6(i) only. The proofs for Theorem 6 (ii)-6(vi) are on the same line, hence omitted. Limiting case of q-beta integral [ 11, p.19 (1.11.7)] is

$$
\frac{1}{\left(q^{x} ; q\right)_{\infty}}=\frac{(1-q)^{-1}}{(q ; q)_{\infty}} \int_{0}^{\infty} t^{x-1}(t q ; q)_{\infty} d_{q} t
$$

Now

$$
\bar{\psi}_{0}(t, \alpha, q)=\frac{1}{(t)_{\infty}} \sum_{n=0}^{\infty} \frac{(t)_{n} q^{2 n^{2}-n+n \alpha}}{(-q ; q)_{2 n}}
$$

Replacing $t$ by $q^{t}$ and $q^{\alpha}$ by $a$, we have

$$
\begin{gathered}
\bar{\psi}_{0}\left(q^{t}, \alpha, q\right)=\frac{1}{\left(q^{t}\right)_{\infty}} \sum_{n=0}^{\infty} \frac{\left(q^{t}\right)_{n} q^{2 n^{2}-n+n \alpha}}{(-q ; q)_{2 n}} \\
=\sum_{n=0}^{\infty} \frac{q^{2 n^{2}-n+n \alpha}}{(-q ; q)_{2 n}\left(q^{n+t}\right)_{\infty}} \\
=\sum_{n=0}^{\infty} \frac{q^{2 n^{2}-n+n \alpha}}{(-q ; q)_{2 n}} \frac{(1-q)^{-1}}{(q ; q)_{\infty}} \int_{0}^{1} w^{n+t-1}(w q ; q)_{\infty} d_{q} w \quad \text { by }(8.1) \\
=\frac{(1-q)^{-1}}{(q ; q)_{\infty}} \int_{0}^{1} w^{t-1}(w q ; q)_{\infty} \sum_{n=0}^{\infty} \frac{q^{2 n^{2}-n}(a w)^{n}}{(-q ; q)_{2 n}} d_{q} w .
\end{gathered}
$$

But

and since $q^{\alpha}=a$,

$$
\bar{\psi}_{0}(0, \alpha, q)=\sum_{n=0}^{\infty} \frac{q^{2 n^{2}-n+n \alpha}}{(-q ; q)_{2 n}},
$$

$$
\bar{\psi}_{0}(0, \alpha, q)=\sum_{n=0}^{\infty} \frac{q^{2 n^{2}-n}(a)^{n}}{(-q ; q)_{2 n}} .
$$

Hence

$$
\bar{\psi}_{0}(0, a w, q)=\sum_{n=0}^{\infty} \frac{q^{2 n^{2}-n}(a w)^{n}}{(-q ; q)_{2 n}} .
$$

By (8.3), (8.2) can be written as

$$
\bar{\psi}_{0}\left(q^{t}, \alpha, q\right)=\frac{(1-q)^{-1}}{(q ; q)_{\infty}} \int_{0}^{1} w^{t-1}(w q ; q)_{\infty} \bar{\psi}_{0}(0, a w, q) d_{q} w,
$$

which proves (i).

\section{Multibasic $q$-Hypergeometric Series Expansions for Generalized Functions}

We shall be using the following summation formula [11, (3.6.7), p. 71] and [17, Lemma 10, p. 57] in writing the multibasic expansions of the generalized functions:

$$
\begin{gathered}
\sum_{k=0}^{\infty} \frac{\left(1-a p^{k} q^{k}\right)\left(1-b p^{k} q^{-k}\right)(a, b ; p)_{k}(c, a / b c ; q)_{k} q^{k}}{(1-a)(1-b)(q, a q / b ; q)_{k}(a p / c, b c p ; p)_{k}} \sum_{m=0}^{\infty} \alpha_{m+k} \\
=\sum_{m=0}^{\infty} \frac{(a p, b p ; p)_{m}(c q, a q / b c ; q)_{m}}{(a p / c, b c p ; p)_{m}(q, a q / b ; q)_{m}} \alpha_{m} .
\end{gathered}
$$

\section{Corollary 1}

Letting $q \rightarrow q^{5}$ and $c \rightarrow \infty$ in (9.1), we have

$$
\begin{gathered}
\sum_{k=0}^{\infty} \frac{\left(1-a p^{k} q^{5 k}\right)\left(1-b p^{k} q^{-5 k}\right)(a, b ; p)_{k} q^{\frac{5 k^{2}+5 k}{2}}}{(1-a)(1-b)\left(q^{5}, a q^{5} / b ; q^{5}\right)_{k} b^{k} p^{\frac{k^{2}+k}{2}}} \sum_{m=0}^{\infty} \alpha_{m+k} \\
=\sum_{m=0}^{\infty} \frac{(a p, b p ; p)_{m} q^{\frac{5 m^{2}+5 m}{2}}}{\left(q^{5}, a q^{5} / b ; q^{5}\right)_{m} b^{m} p^{\frac{m^{2}+m}{2}}} \alpha_{m} .
\end{gathered}
$$




\section{Corollary 2}

Letting $q \rightarrow q^{4}$ and $c \rightarrow \infty$ in (9.1), we have

$$
\begin{gathered}
\sum_{k=0}^{\infty} \frac{\left(1-a p^{k} q^{4 k}\right)\left(1-b p^{k} q^{-4 k}\right)(a, b ; p)_{k} q^{2 k^{2}+2 k}}{(1-a)(1-b)\left(q^{4}, a q^{4} / b ; q^{4}\right)_{k} b^{k} p^{\frac{k^{2}+k}{2}}} \sum_{m=0}^{\infty} \alpha_{m+k} \\
=\sum_{m=0}^{\infty} \frac{(a p, b p ; p)_{m} q^{2 m^{2}+2 m}}{\left(q^{4}, a q^{4} / b ; q^{4}\right)_{m} b^{m} p^{\frac{m^{2}+m}{2}}} \alpha_{m} .
\end{gathered}
$$

\section{Corollary 3}

Letting $q \rightarrow q^{2}$ and $c \rightarrow \infty$ in (9.1), we have

$$
\begin{gathered}
\sum_{k=0}^{\infty} \frac{\left(1-a p^{k} q^{2 k}\right)\left(1-b p^{k} q^{-2 k}\right)(a, b ; p)_{k} q^{k^{2}+k}}{(1-a)(1-b)\left(q^{2}, a q^{2} / b ; q^{2}\right)_{k} b^{k} p^{\frac{k^{2}+k}{2}} \sum_{m=0}^{\infty} \alpha_{m+k}} \\
\quad=\sum_{m=0}^{\infty} \frac{(a p, b p ; p)_{m} q^{m^{2}+m}}{\left(q^{2}, a q^{2} / b ; q^{2}\right)_{m} b^{m} p^{\frac{m^{2}+m}{2}}} \alpha_{m} .
\end{gathered}
$$

\section{Theorem 7}

The generalized functions have the following multibasic hypergeometric series expansion:

(i) $\bar{\psi}_{0}(t, z, q)=\frac{1}{(t)_{\infty}} \sum_{k=0}^{\infty} \frac{\left(1-t q^{6 k-1}\right)\left(1-q^{-4 k+2}\right)(t ; q)_{k-1} q^{2 k^{2}} z^{4 k}}{\left(1-q^{k+2}\right)\left(-q ; q^{2}\right)_{k}\left(-q^{2} ; q^{2}\right)_{k}}$

$\times \phi\left[\begin{array}{c}q, 0: 0,0: t q^{5 k+2}, q^{5 k+5}: \\ q^{k+3}:-q^{2 k+1},-q^{2 k+2}: 0,0:\end{array} ;, q^{2}, q^{5} ; z^{4}\right]$

(ii) $\bar{\psi}_{1}(t, z, q)=\frac{z^{-2}}{(1+q)} \frac{1}{(t)_{\infty}} \sum_{k=0}^{\infty} \frac{\left(1-t q^{6 k-1}\right)\left(1-q^{-4 k+1}\right)(t ; q)_{k-1} q^{2 k^{2}+2 k} z^{4 k}}{\left(1-q^{k+1}\right)\left(-q^{2} ; q^{2}\right)_{k}\left(-q^{3} ; q^{2}\right)_{k}}$

$\times \phi\left[\begin{array}{c}q, 0: 0,0: t q^{5 k+3}, q^{5 k+5}: \\ q^{k+2}:-q^{2 k+2},-q^{2 k+3}: 0,0:\end{array} ;, q^{2}, q^{5} ; q z^{4}\right]$.

(iii) $\bar{\psi}_{0}(t, \alpha, q)=-\frac{1}{(t)_{\infty}} \sum_{k=0}^{\infty} \frac{\left(1+q^{2 k}+q^{4 k}\right)(t ; q)_{k} q^{2 k^{2}-3 k+k \alpha}}{(-q ; q)_{2 k}}$

$\times \phi\left[\begin{array}{l}q, t q^{k}:-q^{2 k+2}: \\ 0,0,0:-q^{2 k+1}: q, q^{2} ; q^{2 k+\alpha-1}\end{array}\right]$.

(iv) $\bar{\psi}_{1}(t, \alpha, q)=-\frac{1}{(t)_{\infty}} \sum_{k=0}^{\infty} \frac{\left(1+q^{2 k}+q^{4 k}\right)(t ; q)_{k} q^{2 k^{2}-k+k \alpha}}{(-q ; q)_{2 k+1}}$

$\times \phi\left[\begin{array}{l}q, t q^{k}:-q^{2 k+2}: \\ 0,0,0:-q^{2 k+3}: q, q^{2} ; q^{2 k+\alpha+1}\end{array}\right]$.

(v) $\bar{\psi}_{2}(t, \alpha, q)=-\frac{1}{(t)_{\infty}} \sum_{k=0}^{\infty} \frac{\left(1-q^{6 k-1}\right)(t ; q)_{k}\left(q ; q^{2}\right)_{k} q^{2 k^{2}-k+k \alpha+1}}{\left(1-q^{2 k+1}\right)\left(q^{4} ; q^{4}\right)_{k}\left(-q ; q^{2}\right)_{k}}$

$\times \phi\left[\begin{array}{c}q, t q^{k}: q^{2 k+1}: \\ \left.0,0,0: q^{2 k+3}: ;, q^{2} ; q^{2 k+\alpha+2}\right] .\end{array}\right.$

(vi) $\bar{\psi}_{3}(t, \alpha, q)=-\frac{1}{(t)_{\infty}} \sum_{k=0}^{\infty} \frac{\left(1+q^{3 k}\right)(t ; q)_{k}(-q ; q)_{k-1} q^{k^{2}-2 k+k \alpha}}{(q ; q)_{k}\left(q ; q^{2}\right)_{k}}$

$\times \phi\left[\begin{array}{c}q, t q^{k}: q^{2 k+2}: \\ 0,0: q^{2 k+1}:\end{array} ;, q^{2} ; q^{k+\alpha-1}\right]$.

\section{Proof of (i)}

We shall give the proof for $\bar{\psi}_{0}(t, z, q)$ in detail, for other functions we shall only give the value of the parameters .

Taking $a=\frac{t}{q}, \quad b=q^{2}, p=q$ and $\alpha_{m}=\frac{\left(q^{5} ; q^{5}\right)_{m}\left(t q^{2} ; q^{5}\right)_{m} z^{4 m}}{\left(q^{3} ; q\right)_{m}\left(-q ; q^{2}\right)_{m}\left(-q^{2} ; q^{2}\right)_{m}}$ in (9.2), we have

$$
\sum_{k=0}^{\infty} \frac{\left(1-t q^{6 k-1}\right)\left(1-q^{-4 k+2}\right)\left(t / q, q^{2} ; q\right)_{k} q^{2 k^{2}}}{(1-t / q)\left(1-q^{2}\right)\left(t q^{2}, q^{5} ; q^{5}\right)_{k}}
$$

$\times \sum_{m=0}^{\infty} \frac{\left(q^{5} ; q^{5}\right)_{m+k}\left(t q^{2} ; q^{5}\right)_{m+k} z^{4(m+k)}}{\left(q^{3} ; q\right)_{m+k}\left(-q ; q^{2}\right)_{m+k}\left(-q^{2} ; q^{2}\right)_{m+k}}=\sum_{m=0}^{\infty} \frac{q^{2 m^{2}}(t ; q)_{m} z^{4 m}}{\left(-q ; q^{2}\right)_{m}\left(-q^{2} ; q^{2}\right)_{m}}$.

The right hand side of (9.5) is equal to

$$
(\mathrm{t} ; \mathrm{q})_{\infty} \bar{\psi}_{0}(t, z, q) \text {. }
$$

The left hand side of (9.5) is equal to

$$
\begin{aligned}
\sum_{k=0}^{\infty} & \frac{\left(1-t q^{6 k-1}\right)\left(1-q^{-4 k+2}\right)(t ; q)_{k-1} q^{2 k^{2}} z^{4 k}}{\left(1-q^{k+2}\right)\left(-q ; q^{2}\right)_{k}\left(-q^{2} ; q^{2}\right)_{k}} \\
& \times \sum_{m=0}^{\infty} \frac{\left(q^{5 k+5} ; q^{5}\right)_{m}\left(t^{5 k+2} ; q^{5}\right)_{m} z^{4 m}}{\left(q^{k+3} ; q\right)_{m}\left(-q^{2 k+1} ; q^{2}\right)_{m}\left(-q^{2 k+2} ; q^{2}\right)_{m}} \\
& =\sum_{k=0}^{\infty} \frac{\left(1-t q^{6 k-1}\right)\left(1-q^{-4 k+2}\right)(t ; q)_{k-1} q^{2 k^{2}} z^{4 k}}{\left(1-q^{k+2}\right)\left(-q ; q^{2}\right)_{k}\left(-q^{2} ; q^{2}\right)_{k}} \\
& \times \phi\left[\begin{array}{c}
q, 0: 0,0: t q^{5 k+2}, q^{5 k+5}: \\
q^{k+3}:-q^{2 k+1},-q^{2 k+2}: 0,0:
\end{array} ; q^{2}, q^{5} ; z^{4}\right],
\end{aligned}
$$

which proves (i).

\section{Proof of (ii)}

Take $a=\frac{t}{q}, \quad b=q, p=q$ and $\alpha_{m}=\frac{\left(q^{5} ; q^{5}\right)_{m}\left(t q^{3} ; q^{5}\right)_{m} q^{m} z^{4 m}}{\left(q^{2} ; q\right)_{m}\left(-q^{2} ; q^{2}\right)_{m}\left(-q^{3} ; q^{2}\right)_{m}}$ in (9.2).

\section{Proof of (iii)}

Letting $q \rightarrow q^{4}, \quad p \rightarrow q^{2}, a=b=1$ and $\alpha_{m}=\frac{(t ; q)_{m}\left(-q^{2} ; q^{2}\right)_{m} q^{m^{2}-2 m+m \alpha}}{\left(-q ; q^{2}\right)_{m}}$ in (9.3).

\section{Proof of (iv)}

Letting $q \rightarrow q^{4}, \quad p \rightarrow q^{2}, a=b=1 \quad$ and $\quad \alpha_{m}=\frac{(t ; q)_{m}\left(-q^{2} ; q^{2}\right)_{m} q^{m^{2}+m \alpha}}{\left(-q^{3} ; q^{2}\right)_{m}}$ in (9.3).

\section{Proof of (v)}

Letting $\quad q \rightarrow q^{4}, \quad p \rightarrow q^{2}, \quad a=\frac{1}{q} \quad, \quad b=q \quad$ and $\alpha_{m}=\frac{(t ; q)_{m}\left(q ; q^{2}\right)_{m} q^{m^{2}+m+m \alpha}}{\left(q^{3} ; q^{2}\right)_{m}}$ in (9.3).

\section{Proof of (vi)}

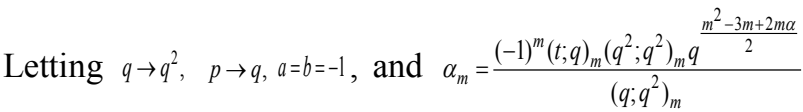
in (9.4). 


\section{Conclusions}

I have given two generalizations of these mock theta functions and shown they are $F_{q}$-functions, so they satisfy the properties of the general class of $F_{q}$-functions. The generalization helps in giving relationship between these functions. Apart from these values, we can give other values to have another set of functions having these properties.

I think these relations may yield interesting results in the theory of partitions.

\section{REFERENCES}

[1] R. P. Agarwal, On integral analogues of certain transformations of well-poised basic hypergeometric series, Quart. J. Math. (Oxford)(2) 4 (1953), 161-167.

[2] R. P. Agarwal, Mock Theta Functions- an analytical point of view, Proc. Nat. Acad. Sci, 64 (1994), 95-107.

[3] G. E. Andrews, The Theory of Partitions, Encycl. Math. and its Appl., vol. 2, Addison- Wesley, Reading, 1976 (Reissued: Cambridge University Press, Cambridge 1985).

[4] G. E. Andrews, Parity in partition identities, Ramanujan Journal (to appear).

[5] G. E. Andrews, $q$-orthogonal polynomials, Rogers- Ramanujan identities, and mock theta functions, preprint.

[6] G. E. Andrews, D. Hickerson, Ramanujan's "lost" notebook VII: The sixth order mock theta functions, Adv. in Math. 89 (1991), 60-105.

[7] R. Askey, Orthogonal polynomials old and new, and some combinatorial connections, Enumeration and Design (D.M. Jackson and S.A Vanstone, eds.) Academic Press, New York (1984), 67-84.

[8] K.Bringmann, K. Hikami, J. Lovejoy, On the modularity of the unified WRT invariants of certain Seifert manifolds, Adv.Appl. Math., to appear.
[9] Youn-Seo Choi, Tenth order mock theta functions in Ramanujan's "lost" notebook IV, Trans. Amer. Math. Soc. 354(2) (2002), 705-733.

[10] N.J. Fine, Basic Hypergeometric Series and applications, Mathematical Surveys and Monographs, Amer. Math. Soc. 27 (1988).

[11] G. Gasper and M. Rahman, Basic Hypergeometric Series vol. 35 of Encyclopedia of Mathematics and its Applications, Cambridge University Press, Cambridge, UK, (1990).

[12] B.Gordon and R.J. McIntosh, Asurvey of classical mock theta functions, preprint.

[13] K. Hikami, Transformation formula of the " $2^{\text {nd" }}$ order mock theta function, Lett. Math. Phys., 75(1) (2006), 93-98.

[14] F.H. Jackson, On $q$-definite integrals, Quart. J. Pure and Appl. Math. 41(1910), 193-203.

[15] F.H. Jackson, Basic Integration, Quart. J. Math.(Oxford) (2), 2 (1951), 1-16.

[16] E. Mortenson, On three third order mock theta functions and Hecke-type double sums, preprint.

[17] E.D. Rainville, Special Function, Chelsea Publishing Company, Bronx, New York (1960).

[18] S. Ramanujan, Collected Paper, Cambridge University Press 1927, reprinted by Chelsea New York, (1962).

[19] Bhaskar Srivastava, Some new mock theta functions, accepted in Math. Sci. Research J.

[20] Bhaskar Srivastava, A study of Bilateral new mock theta functions, (Communicated).

[21] Bhaskar Srivastava, Partial new mock theta functions (Communicated).

[22] C Truesdell, An essay toward a unified theory of special functions, Princeton University Press, Princeton, (1948).

[23] G. N. Watson, The final problem: An account of the Mock theta functions, J. London Math. Soc. 11 (1936), 55-80. 